\title{
Divertor transport study in the Large Helical Device
}

\author{
M. Kobayashi ${ }^{\text {a,* }}$, S. Masuzaki ${ }^{\text {a }}$, M. Shoji ${ }^{\text {a }}$, J. Miyazawa ${ }^{\text {a }}$, \\ T. Morisaki ${ }^{\text {a }}$, N. Ohyabu $^{\text {a }}$, N. Ashikawa ${ }^{a}$, A. Komori ${ }^{\text {a }}$, \\ O. Motojima ${ }^{a}$, and the LHD experimental group ${ }^{a}$ \\ ${ }^{a}$ National Institute for Fusion Science, Oroshi-cho 322-6, Toki 509-5292, Japan \\ Y. Feng $^{\mathrm{b}}$, Y. Igitkhanov ${ }^{\mathrm{b}}$, F. Sardei ${ }^{\mathrm{b}}$ \\ ${ }^{\mathrm{b}}$ Max-Planck-Institut fuer Plasmaphysik, Teilinstitut Greifswald, Euratom \\ Association, 17491 Greifswald, Germany \\ D. Reiter ${ }^{\mathrm{c}}$ \\ ${ }^{\mathrm{c}}$ Institut fuer Plasmaphysik, Forschungszentrum Juelich GmbH, 52425 Juelich, \\ Germany
}

\begin{abstract}
The transport properties of the edge region in LHD have been investigated in order to clarify the divertor/SOL function of the heliotron type device. The momentum loss, mainly through the friction of counter-flows induced by the ergodic field lines, breaks the pressure conservation along flux tubes. This results in no high recycling regime even at high density operation, $\bar{n} \sim 7 \times 10^{19} \mathrm{~m}^{-3}$. The momentum loss is found to be higher than the case of W7-AS. This is because of the higher ratio of perpendicular and parallel transport scale length, $\sim 10^{-4}$, and of the momentum loss due to the friction of counter-flows, which is more effective in the ergodic layer than the island divertor. In the heliotron configuration, a large temperature drop from LCFS to divertor, an order of magnitude, is easily realized due to the long connection length in the ergodic layer. This is certainly a favourable feature for future reactor in terms of reduction of heat power load at the divertor.
\end{abstract}

PACS: 52.55.Hc, 52.55.Rk, 52.25.Fi, 52.65.Pp

* Corresponding author.

Email address: masahiro@nifs.ac.jp (M. Kobayashi). 
JNM keywords: P0600 Plasma Properties, T0100 Theory and Modelling, P0500 Plasma-Materials Interaction

PSI-16 keywords: LHD, 3D edge plasma, Edge modelling, Stochastic boundary

\section{Introduction}

The large Helical Device (LHD) is a heliotron type machine, which has been built to explore more reactor relevant operational regime and to demonstrate an attractive feature of a stellarator [1]. The most remarkable is the recent progress both of the core plasma performance with more than $4 \%$ of beta [2] and the steady state operation with more than one hour [3] and with an input energy of 1.3 GJ [4]. As the core plasma energy content and also the operation duration increase, control of edge plasma transport has become more critical in order to protect the core plasma from an impurity contamination as well as the plasma facing materials from increasing heat load.

In the last few decades since a divertor configuration was first implemented in a tokamaks aiming at reduction of core impurity contamination, it has been extensively investigated both in experimental and theoretical works. It has been shown that the divertor is a key component to realize high performance plasma in the core region.

Following basically the same concept as the tokamaks, LHD is installed with the helical divertor, which can be operated with different magnetic axis positions $\left(R_{a x}\right)$. Its feasibility and the physics of the transport process are, however, not yet fully identified. Different from the poloidal divertor configuration of tokamaks, the magnetic field structure in the edge region consists of the island overlapping region, ergodic layer and divertor legs. This geometrical significance will be most responsible for affecting the edge transport process and would require modifications of the transport picture already developed in tokamaks.

In this paper, the edge transport properties in LHD is analyzed for characterising divertor/SOL function in the heliotron type configuration. Poincare plot of the field line tracing for the configuration of $R_{a x}$ (magnetic axis) = $3.75 \mathrm{~m}$ in LHD is shown in Fig.1. The confinement region with closed flux surfaces is surrounded by ergodic layer of thickness of several to $20 \mathrm{~cm}$, which is created by intrinsically existing magnetic islands at the periphery. The connection length $L_{C}$ in the ergodic layer is of order of kilo meters, and the field lines are connected to the divertor plates through divertor legs. Because of the strong poloidal magnetic component at the legs, the length along the legs from X-point to the plates is only $\sim 2 \mathrm{~m}$. 
As shown in the divertor transport study in W7-AS, the cross field transport starts to play an important role in the islands divertor region [5], which has larger connection length and shorter divertor-to-core distances, compared to tokamaks. From the ratio of $L_{C}$ in the ergodic layer and its width, such condition could be achieved as well in LHD, whereas at the divertor legs, parallel transport will be dominant like in tokamaks. In the followings, the detailed analysis is presented.

\section{Transport in divertor legs : $\mathbf{T}$ drop}

Since the parallel transport is dominating at the divertor legs because of the short length, the standard two-pont model used in tokamaks applies. In the experiments, no strong impurity radiation near the divertor plates is observed, so that no energy sink except at the target surface is assumed along the divertor legs. The energy conduction is given by,

$$
q_{\| \text {cond }}=-\kappa_{0} T^{5 / 2} \frac{d T}{d l}
$$

where $l$ is distance along the legs measured from upstream. Integrating from $\mathrm{X}$-point to divertor plate, it gives,

$$
T_{\text {div }}^{7 / 2}=T_{X}^{7 / 2}-\frac{7}{2} \frac{q_{\| \text {cond }} L_{C}}{\kappa_{0}}
$$

From Bohm condition at the divertor plate, $q_{\| \text {cond }}$ is,

$$
q_{\| \text {cond }}=f_{\text {cond }} \gamma T_{\text {div }} n_{\text {div }} c_{s d}
$$

where $f_{\text {cond }}$ is a fraction of conduction, i.e. $f_{\text {cond }}=q_{\| \text {cond }} / q_{\| \text {total }}$, ranging from 0 to 1 . The measurements of CCD camera and the neutral transport simulation show that there is substantial amount of $H_{\alpha}$ radiation at ergodic layer [6], which gives a certain amount of convection energy. We set thus $f_{\text {cond }}=0.5$. Eqs.(2) and (3) are combined to,

$$
T_{\text {div }}^{2}=\frac{n_{\text {div }} L_{C} f_{\text {cond }}}{C_{1}\left(f_{T}^{7 / 2}-1\right)},
$$

where $f_{T}=T_{X} / T_{d i v}, C_{1}=\frac{2 \kappa_{0} m_{i}^{1 / 2}}{7 \gamma e^{3 / 2}} \approx 5.2 \times 10^{16}$ and $L_{C}=2 \mathrm{~m}$. Eq.(4) is plotted in Fig. 2 for different $f_{T}$ 's together with experimental data of $n_{\text {div }}$ and $T_{\text {div }}$ measured by Langmuir probes at the divertor plate for typical operation 
range in LHD, i.e. line averaged density $\bar{n}_{e}=3 \sim 8 \times 10^{19} \mathrm{~m}^{-3}$ and input power $=4$ to $5 \mathrm{MW}$. It is seen that most of the data is located at the region $T_{X} / T_{d i v}<1.5$. The analysis indicates that there is almost no temperature gradient along divertor legs established. This is attributed to the rather short parallel distance from X-point to divertor plates and also to the open divertor structure, which can not keep recycling neutrals and impurity near the target for radiating and reducing $T_{d i v}$. This is also the reason for no significant density rise at the divertor, i.e. $n_{\text {div }} \leq 1 \times 10^{19} \mathrm{~m}^{-3}$.

\section{Transport in ergodic layer}

\section{$3.1 \perp$ vs. || energy transport}

In the ergodic layer, collisionality is high, i.e. $L_{C} / \lambda_{e e}=L_{C} n /\left(10^{16} T_{e}^{2}\right) \approx 100$ with $n \sim 10^{19} \mathrm{~m}^{-3}$ and $T_{e} \sim 100 \mathrm{eV}$. It can thus be assumed that there is a significant temperature drop along flux tubes (this is confirmed later by the experimental data in section 3.3). Similarly to eqs.(1) and (2) but for uniformly distributed energy input along flux tubes, parallel energy flux is given by,

$$
q_{\|}=\frac{4}{7} \frac{\kappa_{0} T_{u}^{7 / 2}}{L_{C} / 2}
$$

with an assumption that $T_{d i v}^{7 / 2}<<T_{u}^{7 / 2}$, where $T_{u}$ is a temperature at upstream (near LCFS). Using eq.(5), the energy transport time along flux tubes is then expressed as,

$$
\tau_{E \| \mid}=\frac{3 n_{u} T_{u} L_{C}}{2 q_{\|}}=\frac{21 n_{u} L_{C}^{2}}{16 \kappa_{0} T_{u}^{5 / 2}} .
$$

On the other hand, the energy transport time in perpendicular direction in ergodic layer could be given as,

$$
\tau_{E \perp}=\frac{\Delta x^{2}}{2 \chi_{\perp}}
$$

where $\Delta x$ and $\chi_{\perp}$ are radial thickness of the ergodic layer and perpendicular heat conductivity, respectively. For $\Delta x=$ several $\mathrm{cm}$ and $\chi_{\perp}=1.0 \mathrm{~m}^{2} / \mathrm{s}$ (assumed to be anomalous), $\tau_{E \perp}=1 \sim 5 \mathrm{~ms}$. $\tau_{E \|}$ is plotted in Fig.3 as a function of $L_{C}$ for different temperatures together with the range of $\tau_{E \perp}$. It is found that in the range of $100<L_{C}<1000 \mathrm{~m}$, the parallel energy transport time is comparable with the perpendicular one, i.e. $\tau_{E \|} \sim \tau_{E \perp}$, on the other 
hand at $L_{C}>>1000 m$ the perpendicular transport dominates, $\tau_{E \|}>>\tau_{E \perp}$, which corresponds to a confinement region.

Figure 4 (a) and (b) show radial profile of $L_{C}$ and pressure measured by Thomson scattering system at the outer mid-plane, respectively. It is clearly seen that around $\mathrm{R}=4.6 \mathrm{~m}$, where $L_{C}$ becomes lower than $1000 \mathrm{~m}$, the slope of pressure changes, indicating change of transport characteristic.

\subsection{Effect of ergodicity on radial transport}

The sharp change of the slope around $\mathrm{R}=4.6 \mathrm{~m}$ can be qualitatively explained as follows. Provided that the edge region is approximated with a slab geometry with $x$ being radial direction, the radial energy transport could be described with an equation,

$$
-\frac{\partial}{\partial x}\left(-n_{e} \chi_{\perp} \frac{\partial T_{e}}{\partial x}-\frac{5}{2} T_{e} D_{\perp} \frac{\partial n_{e}}{\partial x}\right)=S_{e}
$$

where $S_{e}$ and $D_{\perp}$ are a energy source/sink and particle diffusivity, respectively. Here $\chi_{\perp}$ and $D_{\perp}$ are assumed to be anomalous. For simplicity, let us set $\chi_{\perp}=2.5 D_{\perp}$. Then eq. (8) reads,

$$
-\frac{\partial}{\partial x}\left(-\chi_{\perp} \frac{\partial p_{e}}{\partial x}\right)=S_{e}
$$

here $p_{e} \equiv n_{e} T_{e}$. If all the input power is deposited at $x<x_{0}$ and neglecting energy loss by charge exchange and impurity radiation, eq.(9) can be rewritten for the confinement region $\left(\tau_{E \|}>>\tau_{E \perp}: x_{0} \leq x<x_{1}\right)$ and the intermediate region $\left(\tau_{E \|} \sim \tau_{E \perp}: x_{1} \leq x \leq x_{2}\right)$,

$$
\begin{gathered}
-\frac{\partial}{\partial x}\left(-\chi_{\perp} \frac{\partial p_{e}}{\partial x}\right)=0, \quad\left(x_{0} \leq x<x_{1}\right) \\
-\frac{\partial}{\partial x}\left(-\chi_{e r g} \frac{\partial p_{e}}{\partial x}\right)=\frac{p_{e}}{\tau_{E \|}}, \quad\left(x_{1} \leq x \leq x_{2}\right)
\end{gathered}
$$

where $\chi_{\text {erg }}$ is an effective heat conductivity due to stochasticity of magnetic field. The right hand side of eq.(11) represents energy sink caused by parallel transport in open field lines. Eqs.(10) and (11) give a solution,

$$
p_{e} \propto-\left(x-x_{0}\right), \quad\left(x_{0} \leq x<x_{1}\right)
$$




$$
p_{e} \propto \exp -\frac{x-x_{1}}{\lambda_{e r g}}, \quad\left(x_{1} \leq x \leq x_{2}\right)
$$

where $\lambda_{\text {erg }}=\sqrt{\chi_{\text {erg }} \tau_{E \|}}$. The sharp drop of the pressure around $\mathrm{R}=4.6 \mathrm{~m}$ is considered to come from the exponential decay of eq.(13).

$\lambda_{\text {erg }}$ is plotted in Fig.4 (c) as a function of $\tau_{E \|}$ for different $\chi_{\text {erg }}$ 's. In the ergodic layer, $\tau_{E \|}$ is of order of milli second as discussed in section 3.1, and $\lambda_{\text {erg }}$ is found to be several cm from Fig.4 (b). This means that $\chi_{\text {erg }}=1 \sim 5 \mathrm{~m}^{2} / \mathrm{s}$.

The Kolmogorov length, $L_{K}$, for the present configuration $\left(R_{a x}=3.75 \mathrm{~m}\right)$ was estimated at several tens meters in ref.[7], whereas the mean free path of electron is, $\lambda_{e e} \sim 10 \mathrm{~m}$. If we take collisional case of the Rechester-Rosenbluth's model [8], $\chi_{\text {erg }}$ is,

$$
\chi_{e r g}=D_{F L} v_{t h e} \frac{\lambda_{e e}}{L_{T}},
$$

where $D_{F L}, v_{\text {the }}$ and $L_{T}$ are the diffusion coefficient of magnetic field, electron thermal velocity and the parallel scale length of temperature (parallel decorrelation length, over which a test particle stays inside its original flux tube), $L_{T} \approx 0.5 L_{K} \ln \left[\chi_{\|} D_{F L} /\left(\chi_{\perp} L_{K}\right)\right]$ [9]. We very roughly estimate $D_{F L} \approx \Delta x^{2} / L_{C} \sim 10^{-5} \mathrm{~m}$, at maximum. For the plasma parameter range of the present analysis, $\lambda_{e e} / L_{T} \sim 0.1$ and thus eq.(14) gives $\chi_{e r g} \sim 4 \mathrm{~m}^{2} / \mathrm{s}$. Taking into account the simplification of the model of eq.(14) and rough estimation of $D_{F L}$, the prediction of the model is not so far from the results of the radial transport model. It is also noted that Tokar's model of $\chi_{\text {erg }}$ [10] tends to give lower value than the Rechester-Rosenbluth's one, and the former agrees better with the 3D modelling of the energy transport in ergodic layer of TEXTOR-DED [11]. Although the detailed analysis is still necessary to clarify the effect of the stochasticity on the radial transport, it seems that $\chi_{\text {erg }}$ is not so large in the ergodic layer of this configuration.

\subsection{Effect of cross field transport}

In order to analyze the transport in ergodic layer, the 3D edge transport code, EMC3-EIRENE [12][13], has been implemented on the configuration. Figure 5 (a) and (b) show the electron temperature profile on poloidal plane obtained by the 3D modelling, together with $L_{C}$ profile, respectively, for the case of $R_{a x}=3.75 \mathrm{~m}, n_{u}=2 \times 10^{19} \mathrm{~m}^{-3}, \chi_{\perp}=0.6 \mathrm{~m}^{2} / \mathrm{s}, D_{\perp}=0.3 \mathrm{~m}^{2} / \mathrm{s}$ and the power flow to the SOL, $P_{S O L}$, is $4 \mathrm{MW} . L_{C}$ is resolved up to $10 \mathrm{~km}$, plotted in logarithmic scale. The pattern of long $L_{C}$ region is clearly reflected on the $T_{e}$ profile, appearing as high temperature. This means an energy flow channel 
through long flux tubes, which penetrate closer to LCFS. The radial $T_{e}$ profile measured by the Thomson scattering system is in good agreement with the $3 \mathrm{D}$ code result, as shown in Fig.5 (c), e.g. the decrease at $\mathrm{R}=2.78 \mathrm{~m}$ due to short $L_{C}$ region and the flattening at $\mathrm{R}=2.87 \mathrm{~m}$ caused by the island.

Fig.6 (a) and (b) show the upstream (near LCFS) and divertor plasma parameter as a function of $\bar{n}_{e}$, together with the $3 \mathrm{D}$ modelling results. For the present analysis, impurity transport was not taken into account. The modelling are in reasonable agreement with the experiments except around $\overline{n_{e}} \geq 7 \times 10^{19} \mathrm{~m}^{-3}$, where both temperature and density at the divertor start to decrease, followed by a radiation collapse. It is remarkable that $T_{e}$ decreases from several hundreds $\mathrm{eV}$ at upstream down to a few tens $\mathrm{eV}$ at divertor, i.e. by an order of magnitude. Nevertheless, $n_{\text {div }}$ never exceeds $n_{u}$, and there is no evidence of a high recycling regime like $n_{u} \propto n_{u}^{3}$ and $T_{d i v} \propto n_{u}^{-2}$. It should be noted that the present 3D modelling does not include impurity transport, so that the significant temperature drop across the ergodic layer is not due to the radiation loss.

In order to elucidate the reason for an absence of high recycling behaviour, we introduce cross field transport effect, which was shown to be comparable with parallel transport in ergodic layer in section 3.1. The extended two point model with cross field terms [5] is derived as follows. In the ergodic layer, ratio of cross field and parallel transport scale length is defined as $\beta \equiv \Delta / L_{C}$. The energy (electron plus ion) and momentum transport equations in radial direction are,

$$
\begin{aligned}
& \beta \frac{d}{d x}\left(-\kappa_{0} T^{5 / 2} \beta \frac{d T}{d x}\right)+\frac{d}{d x}\left(-\chi_{\perp} n \frac{d T}{d x}\right)=0, \\
& \beta \frac{d}{d x}\left(m n V_{\|}^{2}+p\right)=-\frac{d}{d x}\left(-D_{\perp} \frac{d}{d x} m n V_{\|}\right)-D_{\perp} \frac{m n \Delta V_{\|}}{\Delta^{2}}
\end{aligned}
$$

where it is assumed that $T_{e}=T_{i}=T$ and energy source/sink due to interaction with neutrals or impurity is negligible. The first terms in eqs.(15) and (16) represent projection of the parallel transport onto the radial direction, $x$, due to the stochastic field lines. The second term on the right hand side of eq.(16) is a momentum loss due to friction between the counter flows which are induced by the ergodic field lines, as found in the 3D modelling. Fig.7 shows Mach number profile in poloidal plane, where yellow and dark blue color indicate flows in positive and negative direction of toroidal angle, respectively. In eq.(16), $\Delta V_{\|}$and $\Delta$ are the relative velocity of two counter flows and the characteristic distance of the opposite flow channels. The boundary condition at the down stream for energy flux is given by,

$$
q_{\|}=\gamma n_{d} T_{d} c_{s d},
$$


and for momentum flux, $-D_{\perp} m n_{d} c_{s d} / \lambda_{\Gamma}$, where $\lambda_{\Gamma}$ is a characteristic decay length of parallel flow in perpendicular direction. At the upstream, perpendicular momentum flux is assumed to be zero. Integrating the eq.(15) and (16) from upstream to downstream, the extended two-point model with cross field terms is obtained as,

$$
\begin{aligned}
T_{u}^{7 / 2} & =T_{d}^{7 / 2}+\frac{7 q_{\|} L_{C}}{2 \kappa_{0}}-\frac{7 \chi_{\perp} n_{u}}{2 \beta^{2} \kappa_{0}}\left(T_{u}-T_{d}\right) \\
p_{u} & =2 p_{d}\left(1+f_{m}\right)
\end{aligned}
$$

where $f_{m}$ is a momentum loss factor,

$$
f_{m}=\frac{D_{\perp}}{2 \beta c_{s d}}\left(\frac{1}{\lambda_{\Gamma}}+\frac{2}{c_{s d} n_{d}} \int \frac{n \Delta V_{\|}}{\Delta^{2}} d x\right)
$$

When $\beta \rightarrow \infty$, the third term on the right hand side of eq.(18) and $f_{m}$ vanish, and the model becomes the standard two-point model in tokamaks.

The results are plotted in Fig.8 for different $f_{m}$ 's, together with the solution obtained by EMC3-EIRENE. Without the cross field terms, the solution shows high recycling behaviour, i.e. $n_{d} \propto n_{u}^{3}$ and $T_{d} \propto n_{u}^{-2}$, which is largely different from the $3 \mathrm{D}$ results. By increasing $f_{m}$ and enhancing the momentum loss, the extended two-point model results approach the 3D code ones. It indicates that the large momentum loss, $f_{m} \sim 9$ i.e. an order of magnitude, suppresses the density increase at downstream, preventing high recycling regime. Compared to the analysis in W7-AS [5], it seems that the momentum loss is larger in LHD. This is because of the smaller $\beta \sim 10^{-4}$, which is $\sim 10^{-3}$ in W7-AS, and the friction between counter flows scattered in the ergodic layer as shown in Fig.7. From eq.(18), it is seen that the large drop of the temperature from LCFS to divertor is due to the long $L_{C}$ in the ergodic layer, compared to other machines. This is a big advantage of the heliotron configuration, where the ergodic layer intrinsically exists at the periphery without additional perturbation coils.

When the density is increased further, $\bar{n} \sim 10^{20} \mathrm{~m}^{-3}$, it was observed that the ion saturation current at the divertor starts to decrease by a factor of 10, and the fraction of impurity radiation reaches $50 \%$ of the total input power [14]. The radiation belt is found to locate around LCFS and rotating in toroidal and poloidal direction. This could be called detachment in the sense that the plasma flux to the divertor significantly decrease, as observed in tokamaks. Since we don't observe strong temperature drop at the divertor prior to the detachment, the physics could be different from that of tokamaks. The mechanism of the detachment is under investigation. 


\section{Conclusion}

The transport properties of the edge region in LHD have been investigated in order to clarify the divertor/SOL function of the heliotron type device. The results of the analysis are summarized as follows.

1. The analysis of $n_{d i v}$ and $T_{d i v}$ measured by Langmuir probes shows that at the divertor legs, no significant temperature drop is expected from X-point to divertor plates. This also means no significant density rise at the divertor plates. Therefore, along the divertor legs, the plasma parameters does not change significantly.

2. In the ergodic layer, at the region of $L_{C}=100 \sim 1000 \mathrm{~m}$, the parallel energy transport time becomes same order of magnitude as the perpendicular one, i.e. $\tau_{E \|} \sim \tau_{E \perp}$. The simple radial transport model shows that the change of transport characteristic appears clearly in the pressure profile across the confinement region $\left(\tau_{E \|}>>\tau_{E \perp}\right)$ to the intermediate region $\left(\tau_{E \|} \sim \tau_{E \perp}\right)$. This is observed in the experimental data as well. From the analysis $f$ the radial transport model as well as the existing models of ergodization effect, it seems that the effective transport coefficient in the ergodic layer, $\chi_{\text {erg }}$, is not so large in the configuration analysed.

3. The momentum loss, mainly through the friction of counter-flows induced by the ergodic field lines, breaks the pressure conservation along flux tubes. This moderate the density rise (temperature drop) at the divertor, resulting in no high recycling regime even at high density operation, $\bar{n} \sim 7 \times 10^{19} \mathrm{~m}^{-3}$. The momentum loss is found to be higher than the case of W7-AS. This is because of the higher ratio of perpendicular and parallel transport scale, $\beta \sim 10^{-4}$, and of the momentum loss due to the friction of counter-flows, which is more effective in the ergodic layer than the island divertor.

4. In the heliotron configuration, a large temperature drop from LCFS to divertor, an order of magnitude, is easily realized due to the long $L_{C}$ in the ergodic layer, without high recycling regime. This is certainly a favourable feature for future reactor in terms of reduction of heat power load at the divertor.

\section{Acknowledgements}

This work was financially supported by NIFS06ULPP530 and NIFS05ULPP530. 


\section{References}

[1] N. Ohyabu et al., Nucl. Fusion 34 (1994) 387.

[2] A. Komori et al., Phys. Plasmas 12 (2005) 056122 and S. Sakakibara et al. to be published in Fusion Science and Technology.

[3] T. Seki et al., Fusion Technol. 40 (2001) 253.

[4] R. Kumazawa et al., Nucl. Fusion 46 (2006) S13.

[5] Y. Feng et al., 10th PET 2005, to be published in Nucl. Fusion.

[6] M. Shoji et al., this conference.

[7] T. Morisaki et al., J. Nucl. Mater. 313-316 (2003) 548.

[8] A.B. Rechester and M.N. Rosenbluth, Phys. Rev. Lett. 40 (1978) 38.

[9] Ph. Ghendrih et al., Plasma Phys. Control. Fusion 38 (1996) 1653.

[10] M.Z. Tokar, Phys. Plasmas 6 (1999) 2808.

[11] M. Kobayashi et al., Nucl. Fusion 44 (2004) S64.

[12] Y. Feng et al., Contr. Plasma Phys. 44 (2004) 57.

[13] D. Reiter et al., Fusion Science and Technology 47 (2005) 172 and www.eirene.de (2004).

[14] J. Miyazawa et al., Nucl. Fusion 46 (2006) 532. 


\section{Figure captions}

Fig.1 :

Poincare plot of field line tracing for the configuration of $R_{a x}=3.75 \mathrm{~m}$. The closed flux surfaces are surrounded by the ergodic layer with the thickness of several to $20 \mathrm{~cm}$. Connection length of the field lines in the ergodic layer is of order of kilo meters. The distance along the divertor legs from X-point to divertor plates is $\sim 2 \mathrm{~m}$.

Fig.2 :

Plot of eq.(4) in $n_{d i v}-T_{d i v}$ space for different $f_{T}=T_{X} / T_{d i v}$. Open circles are probe measurement of $n_{d i v}$ and $T_{d i v}$ at the divertor plates for the operation of $\bar{n}_{e}=3 \sim 8 \times 10^{19} \mathrm{~m}^{-3}$ and input power $=4$ to $5 \mathrm{MW}$.

Fig.3 :

Parallel energy transport time $\tau_{E \|}$, eq.(6), as a function of $L_{C}$ for different $T_{u}$ 's. The range of perpendicular transport time $\tau_{E \perp}$ is also indicated.

Fig.4 :

Radial profile of (a) $L_{C}$ and (b) electron pressure at the outer midplane for $R_{a x}=3.75 \mathrm{~m}$. The pressure was measured by the Thomson scattering system. (c) Decay length of $p_{e}$ in the ergodic layer obtained from eq.(11), $\lambda_{\text {erg }}=$ $\sqrt{\chi_{e r g} \tau_{E \|}}$, as a function of $\tau_{E \|}$ for different $\chi_{e r g}$ 's.

Fig.5 :

(a) $T_{e}$ profile in poloidal plane obtained by EMC3-EIRENE for the case of $R_{a x}=3.75 \mathrm{~m}, n_{u}=2 \times 10^{19} \mathrm{~m}^{-3}, \chi_{\perp}=0.6 \mathrm{~m}^{2} / \mathrm{s}, D_{\perp}=0.3 \mathrm{~m}^{2} / \mathrm{s}$ and $P_{S O L}=$ $4 \mathrm{MW}$. (b) $L_{C}$ profile in poloidal plane. (c) Radial profiles at the inner midplane of $T_{e}$ obtained by the 3D modelling and the experiments for $\bar{n}_{e} \sim 2 \times 10^{1} 9 \mathrm{~m}^{-3}$ and input power $\sim 4 \mathrm{MW}$.

Fig.6 :

Dependence of plasma parameters at upstream (near LCFS) and divertor on $\bar{n}_{e}$, obtained from EMC3-EIRENE and experiments.

Fig.7 :

Mach number profile in poloidal plane obtained by EMC3-EIRENE. The yellow color represents parallel flow in the positive toroidal direction, while the dark blue does the flow in the negative toroidal direction.

Fig.8 :

Solution of the extended two-point model, eqs.(17) - (20), together with the $3 \mathrm{D}$ results. (a) downstream density $n_{d}$, (b) solid lines for $T_{d}$, broken lines for $T_{u}$. Black : standard 2PM, blue : extended 2PM with $f_{m}=0$, red : extended $2 \mathrm{PM}$ with $f_{m} \sim 2$, green : extended $2 \mathrm{PM}$ with $f_{m} \sim 9$. The results of EMC3EIRENE are indicated by lines with circles. 


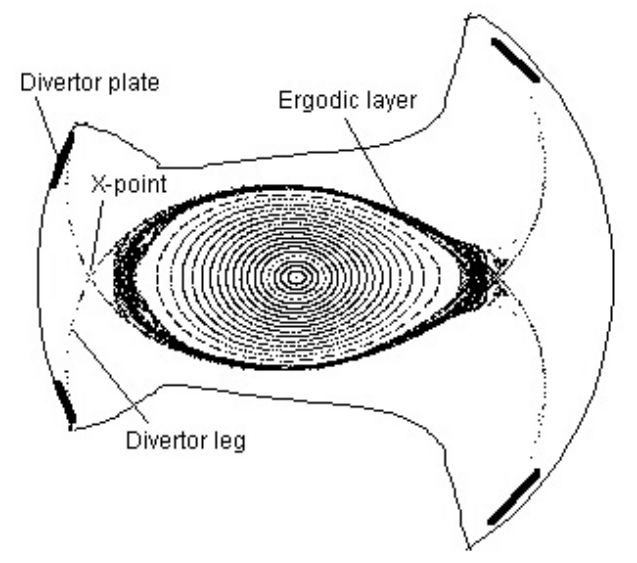

Fig. 1. 


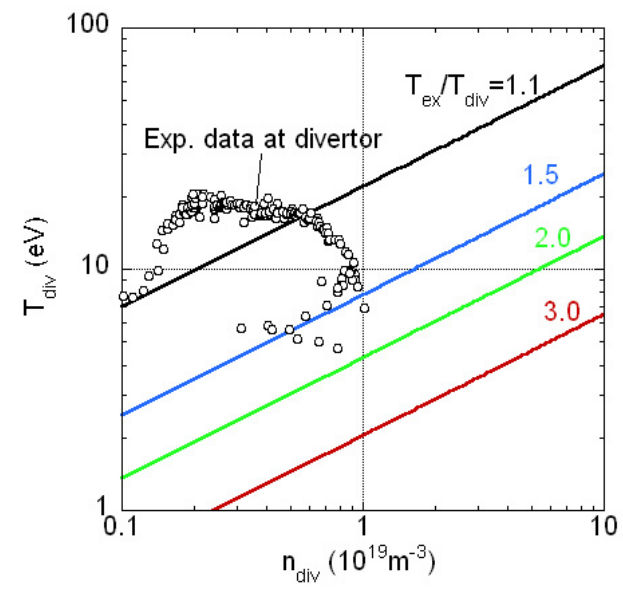

Fig. 2. 


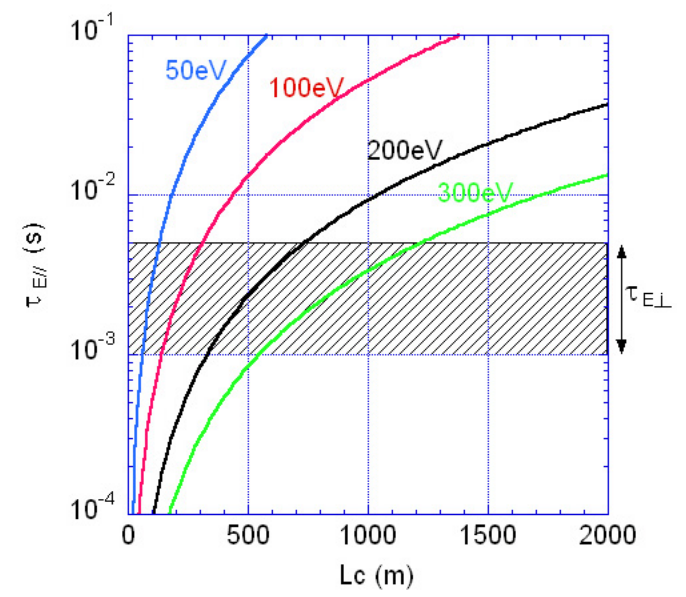

Fig. 3. 

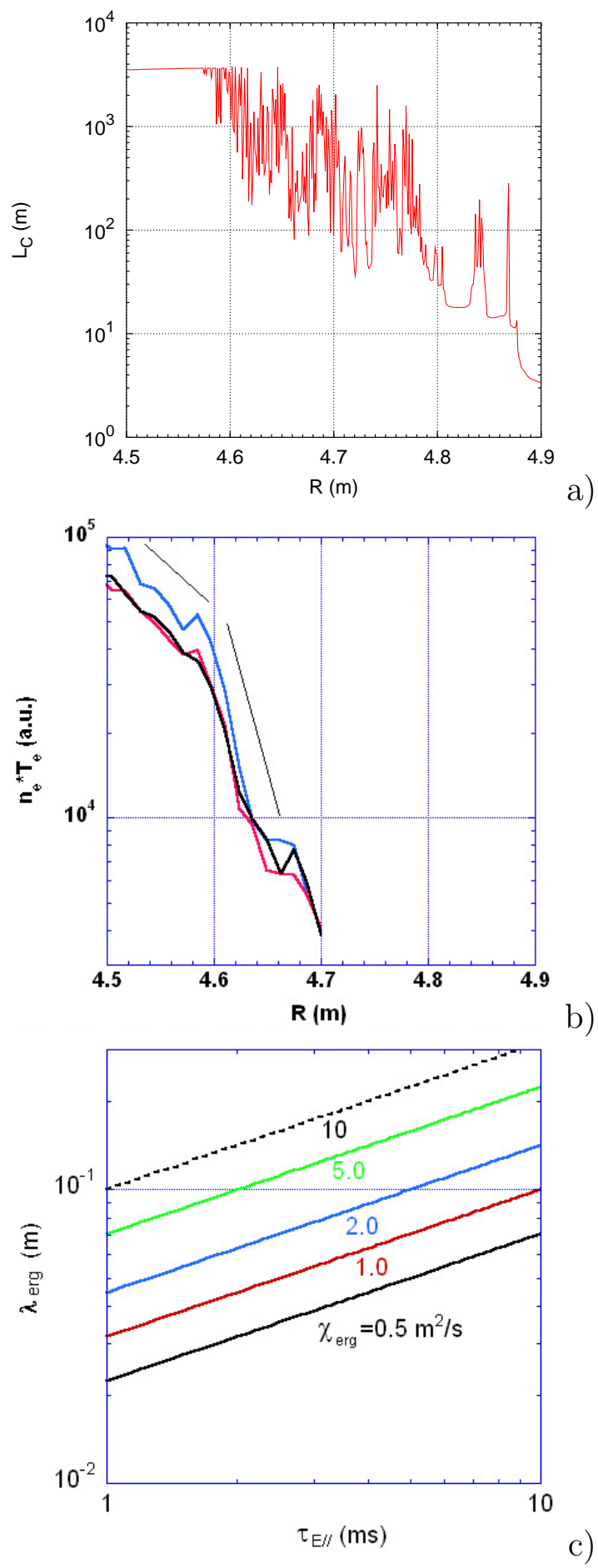

Fig. 4. 

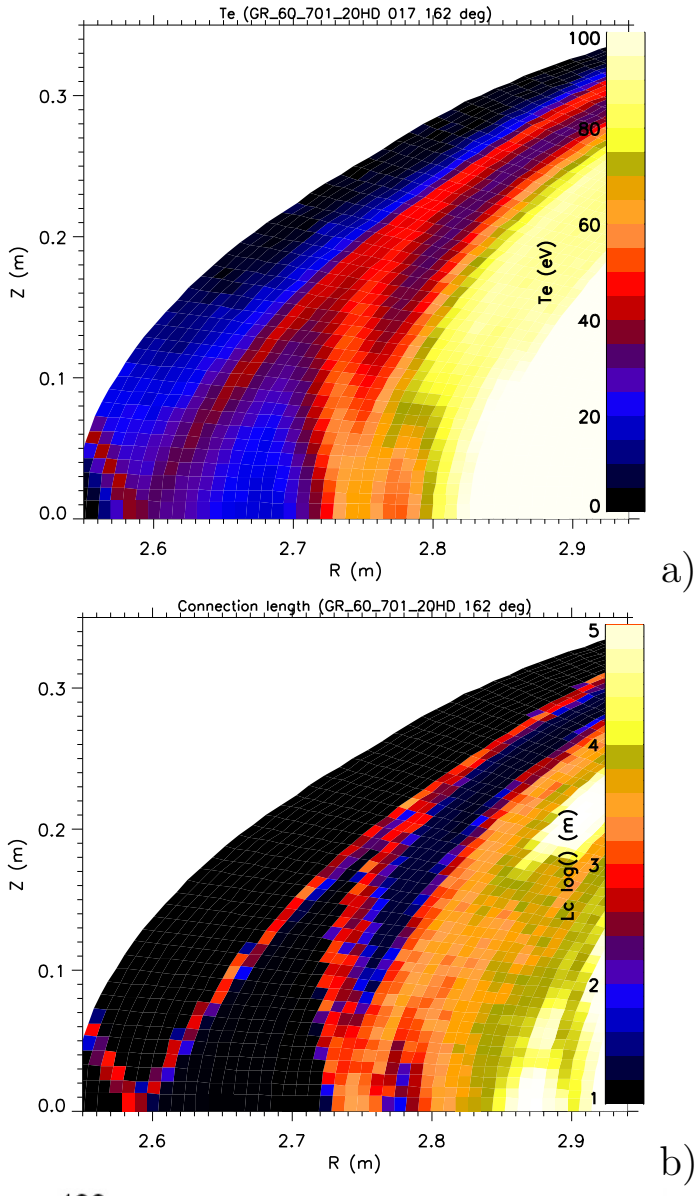

b)

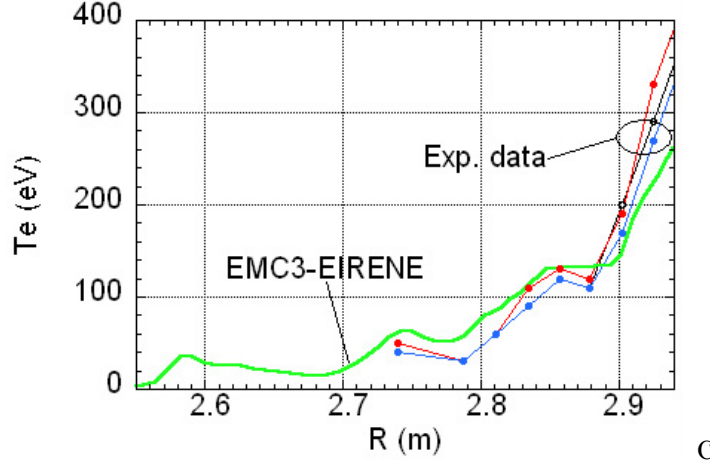

Fig. 5. 


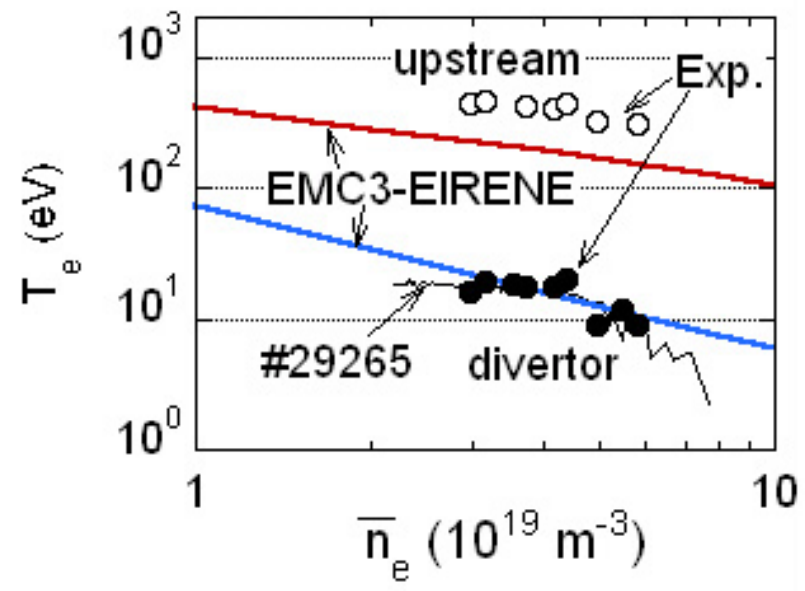

a)

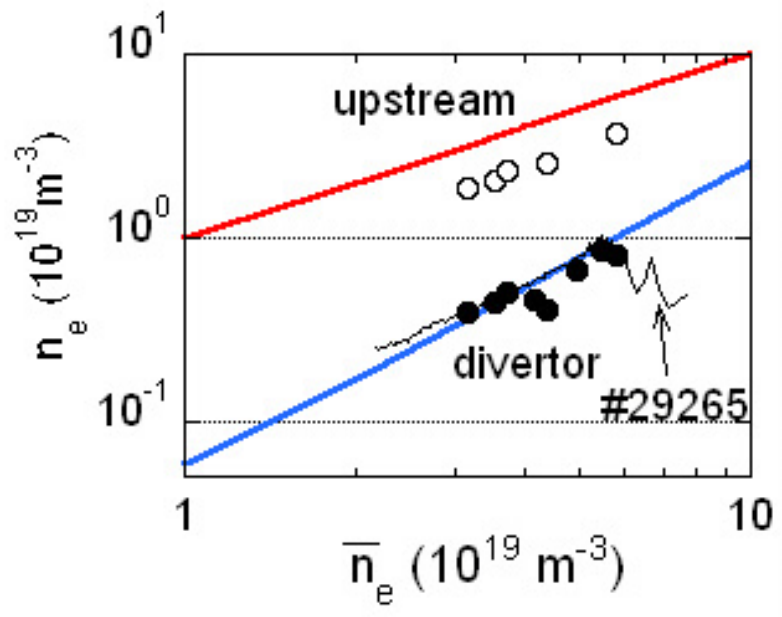

b)

Fig. 6. 


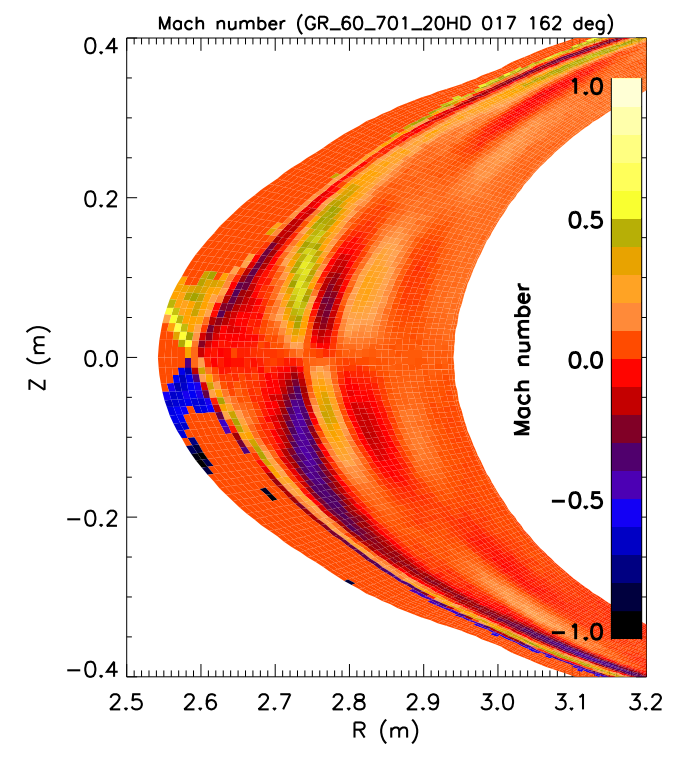

Fig. 7. 

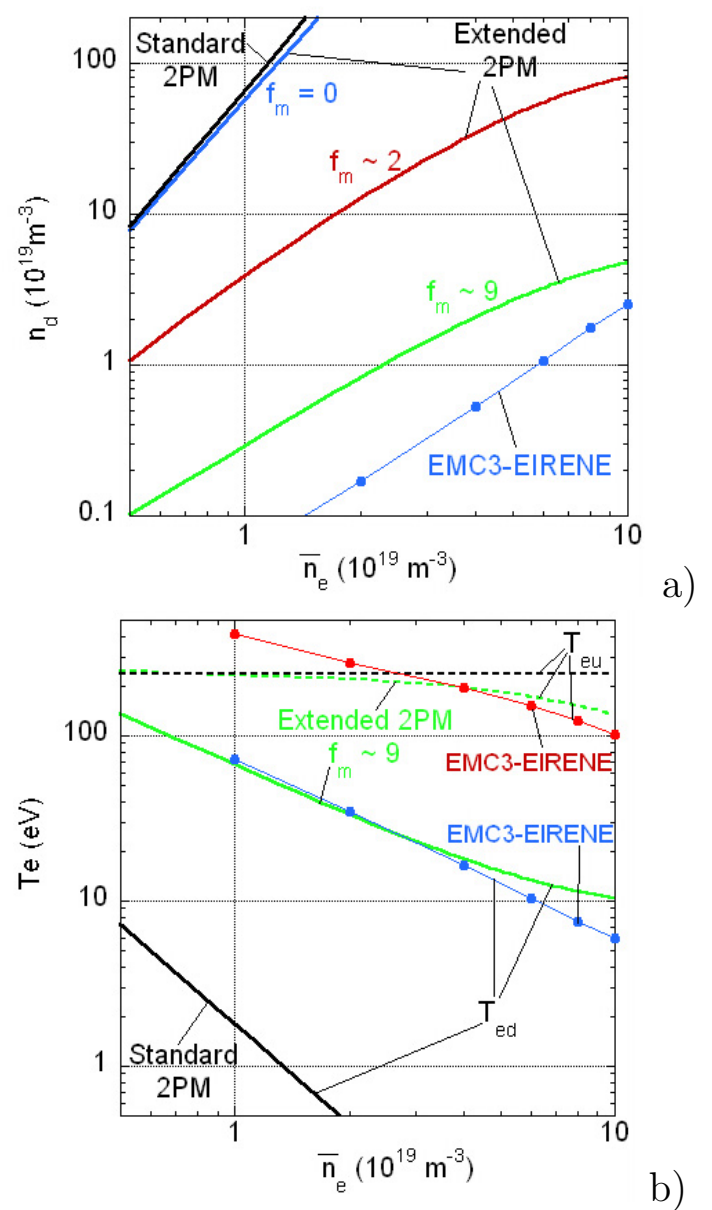

b)

Fig. 8. 